\title{
A procedure for dual simple t-cannulation in the small intestine of pigs using a single right flank laparotomy
}

\author{
A.L. Petry, S.A. Gould, and J.F. Patience* \\ Department of Animal Science, Iowa State University, Kildee Hall, 806 Stange Road, Ames, IA 50011, USA; jfp@iastate.edu
}

Received: 3 June 2020 / Accepted: 6 October 2020

(C) 2020 A.L. Petry et al.

\section{RESEARCH ARTICLE}

\begin{abstract}
Singular cannulation in the small intestine of pigs is a common methodology for studying nutrient digestibility, delivering compounds into the gastrointestinal tract, or repeated tissue and digesta sampling. In that respect, it is an important tool for nutritionists and researchers. However, there is a dearth of detailed methodologies describing multiple intestinal cannulations using modern techniques. The objective of this experiment was to develop a single right flank laparotomy technique that allowed for imsertion of multiple cannulas in the small intestine, with minimal variation, and allowed for externalisation of both cannulas on the same lateral side as the laparotomy. Thirty gilts (L337 $\times$ Camborough) with an initial body weight of $30.2 \pm 0.78 \mathrm{~kg}$ underwent the procedure. Each gilt was equipped with a simple t-cannula in the terminal ileum, approximately $10 \mathrm{~cm}$ cranial to the ileocecal valve, and a second $\mathrm{t}$-cannula in the jejunum $240 \mathrm{~cm}$ distal from where the duodenum is visually posterior to the transverse colon. The procedure used hallmarks of commonly implemented terminal ileal cannulation techniques, but modified the laparotomy location and cannula externalisation sites and used a novel approach for determining the more proximal cannula location to mitigate the need of a second laparotomy. Gilts were allowed to recover for a minimum of $7 \mathrm{~d}$ and were used for an average of 67 days in subsequent experimental trials. Cannula longevity was from 30 to $73 \mathrm{~kg}$ of body weight. Pigs were necropsied for surgical site gross examination and small intestine measurements at the end of the experiment. The jejunal cannula had a mean placement of $298.90 \pm 9.96 \mathrm{~cm}$ distal to the pyloric sphincter with a coefficient of variation of $3.33 \%$. Hence, this procedure provided a single laparotomy technique for obtaining digesta from multiple locations of the small intestine, with minimal variation in proximal cannula placement.
\end{abstract}

Keywords: digestibility, ileal cannulation, jejunal cannulation, swine, t-cannula

\section{Introduction}

Collecting contents from the gastrointestinal tract is a vital part of many nutrition and gastrointestinal physiology studies. Digesta can be collected by way of the slaughter technique, rectal anastomosis, or with intestinal cannulation methods. The slaughter technique allows for collection from multiple sites of the gastrointestinal tract, but sequential sampling is unachievable, sample quantity is limited, and it requires a greater number of animals (Albin et al., 1999; Li et al., 2019). Rectal anastomosis allows for multiple collections but involves removal or bypass of the large intestine and prevents collection from multiple gastrointestinal sections (Green et al., 1987). In recent years, the simple t-cannulation technique has emerged as a common technique in swine nutrition for evaluating the ileal digestibility of nutrients (Gutierrez et al., 2016). This methodology has been used for studying gastrointestinal content rate of passage, a port to deliver compounds into the intestine, and for live endoscopic tissue sampling (Beale et al., 2018; Newman et al., 2016).

Compared to the aforementioned collection methods, simple t-cannulation is a less invasive method that sustains a more normal physiological state and has been associated with reduced variation in placement and sampling (Knabe $e t$ 
al., 1989). Procedural methods for simple ileal t-cannulation have been reported in young piglets, growing pigs and gestating sows (Gargallo and Zimmermann, 1980; Stein et al., 1998; Walker et al., 1986). These methods, while differing in the type and make of cannulas used, have similar surgical procedures and characteristics, and utilise a single right flank laparotomy. However, one limitation among these studies is that the methodology only considers the implementation of one cannula, limiting digesta collection to a single point in the intestine.

Although scarce, procedures that used multiple $t$-cannulas or intestinal fistulas have been reported, but these studies were published prior to the modern $\mathrm{t}$-cannulation methodologies utilised today, involved more invasive surgical techniques, required multiple midline laparotomies or a cranial to midabdominal laparotomy, and some externalisation of the cannulas on both sides of the pig (Braude et al., 1976; Furuya et al., 1974; Kik et al., 1988). As such, techniques for equipping pigs with multiple $t$-cannulas that more closely align to the procedures utilised today are warranted. Therefore, the experimental objective was to develop a single right flank laparotomy technique that permitted the insertion of multiple cannulas in the small intestine with minimal variation and allowed for externalisation of both cannulas on the same lateral side as the laparotomy. It was hypothesised that, by using two anatomical landmarks, i.e. the duodenum suspensory ligament and the point where the duodenum is posterior to the transverse colon, the standard single ileal $t$-cannulation procedure could be expanded to position a second $t$-cannula at the mid-point of the jejunum. This would support research that among other things, studied the progress of digestion at two points in the small intestine.

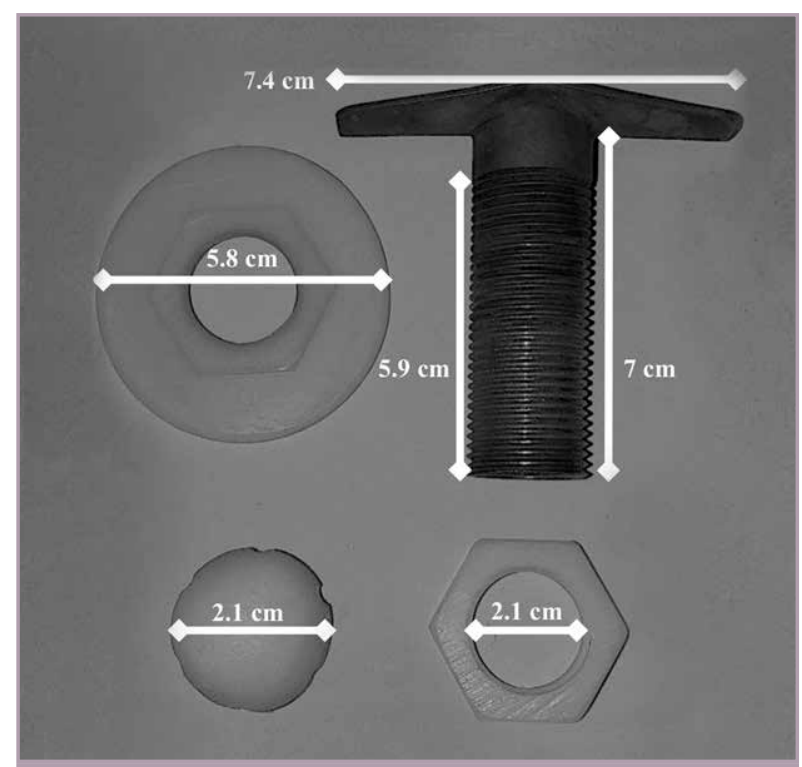

Figure 1. Ileal t-cannula.

\section{Materials and methods}

All experimental procedures adhered to guidelines for the ethical and humane use of animals for research according to the Guide for the Care and Use of Agricultural Animals in Research and Teaching (FASS, 2010), and were approved by the Iowa State University Institutional Animal Care and Use Committee (2-18-8705-S). Thirty gilts (L337 × Camborough) with an initial body weight of $30.2 \pm 0.8 \mathrm{~kg}$ underwent the dual $\mathrm{t}$-cannulation procedure using the methodology described below.

\section{Description of t-cannulas}

The ileal simple t-cannula (Kremer Precision Machine, Shumway, IL, USA) was made of titanium (Figure 1), and had a wing shaped flange with tapered corners that were 7.4 $\mathrm{cm}$ long and $2.8 \mathrm{~cm}$ wide at the widest point, and a tubular barrel with an outer diameter of $2 \mathrm{~cm}$ and an inner diameter of $1.8 \mathrm{~cm}$. The barrel was $7 \mathrm{~cm}$ long and the external threaded surface was $5.9 \mathrm{~cm}$ in length. A plastic washer with an outer diameter of $5.8 \mathrm{~cm}$ and a central thread hole (2.1 cm diameter) was used to secure the $\mathrm{t}$-cannula to the body wall. A second plastic washer with a central thread hole (2.1 cm diameter) was used, and a polyvinyl chloride cap with internal threads and an internal diameter of 2.1 $\mathrm{cm}$ was used to close the $\mathrm{t}$-cannula.

The jejunal simple t-cannula (Kremer Precision Machine) was made of stainless steel (Figure 2) and had a wing shaped flange with tapered corners that were $6.2 \mathrm{~cm}$ long and 2.2 $\mathrm{cm}$ wide at the widest point, and a tubular barrel with an outer diameter of $1.7 \mathrm{~cm}$ and an inner diameter of 1.6 $\mathrm{cm}$. The barrel was $6.3 \mathrm{~cm}$ long and the external threaded surface was $3.2 \mathrm{~cm}$ in length. A stainless-steel washer with

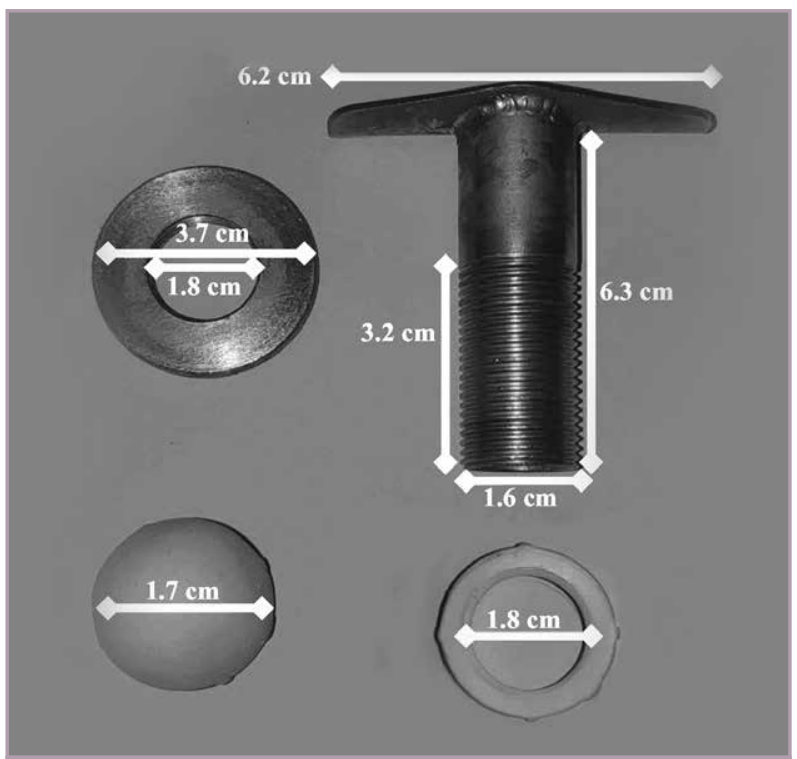

Figure 2. Jejunal t-cannula. 
an outer diameter of $3.7 \mathrm{~cm}$ and a central thread hole $(1.8$ cm diameter) was used to secure the t-cannula to the body wall. A second plastic washer with a central thread hole diameter of $1.8 \mathrm{~cm}$ was used and a polyvinyl chloride cap with internal threads and an internal diameter of $1.7 \mathrm{~cm}$ was used to close the t-cannula.

\section{Surgical preparations and monitoring}

Approximately 17 hours prior to surgery, pigs were weighed and fasted. Pigs were anaesthetised approximately 15 minutes before the start of surgery with a $1.5 \mathrm{ml}$ intramuscular injection from a $5 \mathrm{ml}$ vial of tiletamine $\mathrm{HCl}$ and zolazepam $\mathrm{HCl}$ (Telazol, $100 \mathrm{mg} / \mathrm{ml}$; Zoetis Inc., Kalamazoo, MO, USA) diluted with $2.5 \mathrm{ml}$ of ketamine $\mathrm{HCl}$ (Ketaset, $100 \mathrm{mg} / \mathrm{ml}$; Zoetis Inc.) and $2.5 \mathrm{ml}$ of xylazine $\mathrm{HCl}$ (AnaSed LA, $100 \mathrm{mg} / \mathrm{ml}$; VET one, Boise, ID, USA). After being anesthetised, an approximate $25 \times 25 \mathrm{~cm}$ area of the right dorsal flank was shaved, and cleaned with a sponge and warm water. The surgical area was further cleansed by scrubbing the incision site and moving outward in a circular motion, alternating with iodine and isopropyl alcoholsoaked gauze. Pigs were positioned on the surgical table in left lateral recumbency, and an anaesthesia mask was placed around the snout and secured. Oxygen was supplied at $1.5 \%$ of end-tidal volume, and anaesthesia was maintained with $3 \%$ isoflurane through a closed anaesthetic circle system. Once full anaesthesia was achieved, determined by eye reflex, the rate of isoflurane was reduced to $1.5 \%$. All surgeries were performed using aseptic techniques. Members of the surgical team scrubbed, gowned and gloved before each surgery. Surgical instruments and supplies were either purchased sterile or sterilised in an autoclave at $120{ }^{\circ} \mathrm{C}$ at 19 psi for 30 minutes. Respiration rate, rectal temperature, pulse, and $\mathrm{O}_{2}$ saturation were measured every 15 minutes from the time of sedation until the pig was alert and responsive to stimuli.

\section{Laparotomy}

The pig was draped with a sterile impervious sheet, and the sheet was secured with Backhaus towel clamps. An approximate $20 \mathrm{~cm}$ by $20 \mathrm{~cm}$ area of drape was removed to expose the surgical area. An incision, approximately 12 $\mathrm{cm}$ in length, of the subcutaneous tissue and cutaneous muscular layer was made approximately $3 \mathrm{~cm}$ distal and parallel to the last rib (Figure 3A). The incision was continued by manual separation of the external and internal abdominal oblique muscles and transverse abdominal muscle, exposing the peritoneum. Forceps were used to retract the peritoneum laterally from the abdominal cavity, and a small incision in the centre of the exposed peritoneum was made using a scalpel. The peritoneum was then cut to the length of the initial incision.

\section{Ileal t-cannulation}

Immediately caudal to the incision, the caecum was identified, and the ileocaecal junction was located (Figure 3B). A $15 \mathrm{~cm}$ section of the terminal ileum, approximately 3 $\mathrm{cm}$ anterior to the ileocecal ligament, was isolated from the abdominal cavity. Laparotomy sponges, soaked with sterile saline $(9 \% \mathrm{NaCl})$, were placed around the isolated section of intestine. A purse string suture, two-thirds of the length of the flange of the ileal t-cannula, was placed on the long axis of the ileum submucosa using 2-0 braided polyglactin 910 suture (Ethicon US, Somerville, NJ, USA) while leaving two extremes without notch. A $4 \mathrm{~cm}$ enterotomy was made between the purse-string sutures. The flanges of the $t$-cannula were inserted into the enterotomy, and the two suture extremes were pulled closed to secure the enterotomy around the t-cannula (Figure 3C). A second purse string was placed in the submucosa as reinforcement to the first purse-string. To exteriorise the ileal $t$-cannula, a $3 \mathrm{~cm}$ stab incision was made behind the last rib and dorsal to the initial incision. The incision was positioned to ensure the flanges of the $\mathrm{t}$-cannula did not interfere with the musculature of the hind limb or affect the kidney and allowed adequate space to exteriorise the second $t$-cannula (Figure 3D). The barrel of the $\mathrm{t}$-cannula was exteriorised through the incision, caution was used to ensure the intestine was not twisted, and the body wall pulled down the length of the barrel until secure. The t-cannula flanges were palpated, ensuring that the small intestine was not pinched between the t-cannula and the body wall. The barrel of the t-cannula was cleaned, and a plastic washer was placed on the barrel threads until it reached the base of the skin, to secure the t-cannula in place.

\section{Jejunal t-cannulation}

Cranial to the laparotomy incision, the abdominal cavity was palpated for the pyloric sphincter, and the small intestine was palpated caudally until the duodenum was visualised to be posterior to the transverse colon. From that point, a $240 \mathrm{~cm}$ sterile cotton string was used to caudally measure the small intestine (Figure 3E). Care was taken to place the removed jejunum back into the abdomen approximately every $50 \mathrm{~cm}$. After measuring, a $15 \mathrm{~cm}$ section of medial jejunum was isolated from the abdominal cavity using saline soaked laparotomy sponges. The same procedure described for placing and securing the ileal t-cannula was used for the jejunal $t$-cannula (Figure 3F). The jejunal t-cannula was externalised caudal to the last rib and ventral and anterior to the ileal t-cannula using the same technique described for the ileal t-cannula (Figure 3G). 

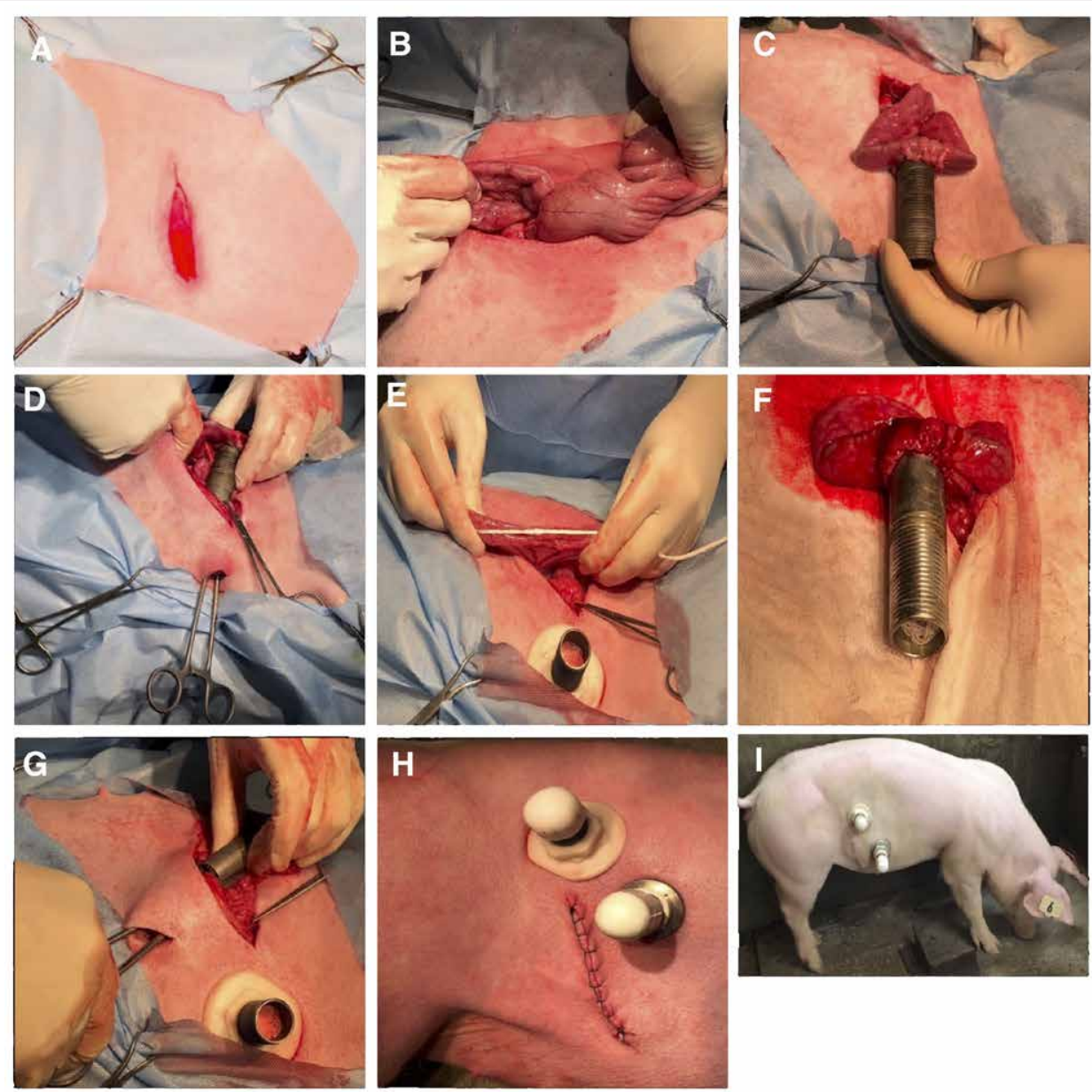

Figure 3. Visual representation of surgical procedure and cannula placement. (A) 12-cm incision of the subcutaneous tissue and cutaneous muscle layer made approximately $3 \mathrm{~cm}$ distally and parallel to the last rib. (B) locating the terminal ileum from the ileocecal junction. (C) ileal t-cannula after reinforcement purse string was placed and before externalisation of the t-cannula. (D) ileal $t$-cannula exteriorisation through a $3 \mathrm{~cm}$ stab incision. (E) depiction of measuring the jejunum $240 \mathrm{~cm}$ distal from where the duodenum is visually posterior to the transverse colon with the use of a string. (F) jejunal t-cannula after reinforcement purse string was placed and before externalisation of the t-cannula. (G) jejunal t-cannula exteriorisation caudal to the last rib and anteriorly to the ileal t-cannula. $(H)$ the right side of the pig after t-cannula placements and laparotomy closure. (I) a gilt $10 \mathrm{~d}$ post-surgery after suture removal.

\section{Laparotomy closure}

The peritoneum was closed with 2-0 polyglactin 910 suture in a simple continuous pattern. Similarly, the transverse abdominal muscle was closed in the same manner with the same suture. The internal and external abdominal oblique muscles were closed together with 0 polyglactin suture (Ethicon US) in a simple continuous pattern. After closure, the external abdominal oblique was injected with $1 \mathrm{ml}$ of ceftiofur crystalline free acid (Excede, $100 \mathrm{mg} / \mathrm{ml}$; Zoetis Inc.). The cutaneous muscle and skin were closed together with 0 polyglactin using the Ford interlocking pattern. A picture of the closed incision in relation to the position of the cannula is depicted in Figure 3H. Flunixine meglumine (Banamine, $50 \mathrm{mg} / \mathrm{ml}$; Merck Animal Health, Madison, NJ, USA) was administered intramuscularly after closure at a dose of $2 \mathrm{ml}$ per $45 \mathrm{~kg}$ of body weight.

\section{Postsurgical care and experimental collections}

Once recovered from anaesthesia, pigs were housed in individual pens $(1.8 \times 1.9 \mathrm{~m})$ equipped with a nipple drinker on a partially slatted concrete floor. Pigs were allowed to recover for a minimum of seven days. Pigs were fed a standard corn-soya bean meal based growing diet, that met dietary requirements for growing pigs, during the recovery period. Pigs were fed $50 \mathrm{~g}$ at 24 hours after surgery, and feed allowance was gradually increased to a maximum of 2.7 times their maintenance energy requirement by day three of recovery (NRC, 2012). Flunixine meglumine was administered intramuscularly the day after surgery at a dose of $2 \mathrm{ml}$ per $45 \mathrm{~kg}$ body weight, and a $1 \mathrm{ml}$ intramuscular injection of ceftiofur crystalline free acid was administered once a day for three days post-operative. The skin around the $\mathrm{t}$-cannulas was washed daily with warm water, and 
a zinc oxide lanolin-based cream was applied around the cannulas as needed to reduce skin irritation. Triple antibiotic ointment was applied to the incision for three days post-operative to prevent infection, and sutures were removed seven to 10 days post-operative. Figure 3I depicts a gilt 10 days post recovery with a healed incision.

After recovery, pigs were utilised in subsequent experimental trials. In these trials, ileal digesta was collected by removing the cap from the t-cannula and attaching a $17.8 \times 9.5 \mathrm{~cm}$ sterile, low density, polyethylene collection bag with a capacity of $207 \mathrm{ml}$ (Nasco; Fort Atkinson, WI, USA) to the outer part of the t-cannula barrel, with an auto locking cable tie. These bags were removed as soon as they were filled with digesta or at least once every hour. Jejunal digesta was collected in a similar manner, but only one bag per hour for six hours post feeding. The ileal cannula was used as an intestinal access point for administration of barium sulphate into the large intestine in one study.

\section{Post-mortem examination}

At the termination of experimental use, gilts were necropsied for gross examination of the surgical site and small intestine measurements. The small intestine, from the pyloric sphincter to the ileocaecal junction, was removed from the abdominal cavity, and clamps were placed at the beginning and end of the jejunal and ileal $t$-cannula locations. The length of intestine was measured from the pyloric sphincter to the jejunal t-cannula, from the jejunal $\mathrm{t}$-cannula to the ileal $\mathrm{t}$-cannula, from the ileal $\mathrm{t}$-cannula to the ileocecal junction, and from the pyloric sphincter to the ileocaecal junction.

\section{Results}

Dual t-cannulation was performed successfully on 30 gilts; from initial laparotomy to closure, the mean surgical procedure length was 68 minutes. Pigs recovered from anaesthesia, approximately 3 hours post-surgery, and normal behaviour was observed by the following day. Two mortalities occurred during the recovery period; for one the cause was unknown, and the other was due to apparent twisting of the jejunal cannula. Similarly, one gilt was euthanised during the recovery period for twisting of the jejunal cannula. To alleviate torsion of the jejunal cannula, an additional support washer, with a diameter of $6 \mathrm{~cm}$, was placed against the skin on the remaining gilts, and implemented in all subsequent procedures using this technique. No other incidents of jejunal cannula torsion occurred after the implementation of the additional washer.

Feed intake was gradually increased during the first three days post-operative to 2.7 times the maintenance energy requirement, but most pigs did not achieve full intake until five days after recovery. T-cannula longevity, defined as the duration when continuous sample collection could occur, was 67 days or from 30 to $72 \mathrm{~kg}$ mean body weight. In this instance, the diets were based on corn and soybean meal or corn, soybean meal and 30\% corn bran, and contained between 12 and $25 \%$ total dietary fibre. No difficulty in sample collection was observed and flow through the cannulae was unimpaired. On average, each gilt had digesta collected from the jejunal and ileal cannula on nine and 12 separate days, respectively. At experimental termination, pigs had a mean body weight of $73.1 \pm 4.5$ $\mathrm{kg}$ with a coefficient of variation of $6.1 \%$ (Table 1). Gross examination of each $\mathrm{t}$-cannulation site revealed the small intestine fused well with the peritoneum and body wall.

The mean distance from the pyloric sphincter to the ileocaecal junction was $1,613.5 \mathrm{~cm}$ with a coefficient of variation of $2.95 \%$ (Table 1). The mean distance from the pyloric sphincter to the jejunal t-cannula was $298.9 \mathrm{~cm}$ with a coefficient of variation of $3.33 \%$. The distance from the jejunal t-cannula to the ileal t-cannula was $1,361.18 \mathrm{~cm}$ with a coefficient of variation of $3.50 \%$.

Table 1. Descriptive statistics of initial and final body weight and small intestine measurements of 28 pigs surgically fitted with a t-cannula in the jejunum and terminal ileum. ${ }^{1}$

\begin{tabular}{|c|c|c|c|c|c|c|c|}
\hline \multirow[t]{2}{*}{ Item } & & \multicolumn{6}{|c|}{ Descriptive statistics } \\
\hline & & Mean & SEM & SD & Min & Max & $\mathrm{CV} \%$ \\
\hline \multirow[t]{2}{*}{ Body weight, kg } & Initial & 30.2 & 0.15 & 0.78 & 28.5 & 31.7 & 2.6 \\
\hline & Final & 73.1 & 0.85 & 4.49 & 68.1 & 82.4 & 6.1 \\
\hline \multirow[t]{4}{*}{ SI measurements, $\mathrm{cm}$} & pyloric sphincter to ileocecal junction & $1,613.5$ & 9.0 & 47.6 & $1,451.2$ & $1,722.1$ & 2.95 \\
\hline & pyloric sphincter to jejunal t-cannula & 298.9 & 1.9 & 10.0 & 266.3 & 302.3 & 3.33 \\
\hline & jejunal t-cannula to ileal t-cannula & $1,361.2$ & 9.1 & 47.6 & $1,150.0$ & $1,407.2$ & 3.50 \\
\hline & ileal t-cannula to ileocecal junction & 13.4 & 0.4 & 2.2 & 14.9 & 25.4 & 17.06 \\
\hline
\end{tabular}

${ }^{1} \mathrm{CV} \%$ = coefficient of variation; $\mathrm{SD}=$ standard deviation; $\mathrm{SEM}=$ standard error of the mean; $\mathrm{SI}=$ small intestine. 


\section{Discussion}

There is a dearth of literature regarding methods for sequentially collecting digesta from multiple gastrointestinal locations within the same pig. Previously reported multiple $\mathrm{t}$-cannulation methods have used more invasive and less efficient surgical techniques compared to the commonly implemented, modern singular cannulation methods. The experimental objective was to develop a single right flank laparotomy technique that allowed for the implementation of multiple cannulas in the small intestine, with minimal variation, while externalising both cannulas on the same lateral side as the laparotomy. The procedure described herein was a modified technique of a previously reported distal ileal t-cannulation techniques in pigs (Gargallo and Zimmermann, 1980; Stein et al., 1998). Modifications included the implementation of a novel technique for identifying and measuring the small intestine for the proximal cannula placement, adjusting the location and extending the length of the initial right flank laparotomy, and altering the location of cannula externalisation.

In ileal cannulation procedures, the placement of the ileal $t$-cannula is measured cranially from the ileocaecal junction, but, due to the length and arrangement of the small intestine, this approach is not feasible for more proximal t-cannula placement. Therefore, a slightly larger right flank laparotomy was necessary to palpate the abdominal wall for the duodenum suspensory ligament to measure distally from the pyloric sphincter. However, the position of the transverse spiral colon, when a gilt is in lateral recumbency, rendered it impractical to measure distally from the pyloric sphincter to the desired location of t-cannula placement. This may explain why previously reported multiple $\mathrm{t}$-cannulation procedures utilised a cranial to midabdominal midline laparotomy to access the intestine (Braude et al., 1976; Furuya et al., 1974). However, midline laparotomies have been associated with a greater risk of post-surgical infections, longer recovery periods and increased surgical time (Swindle and Smith, 2015).

To mitigate the need for a midline laparotomy, the technique reported herein measured caudally from where the duodenum was visually distal to the transverse colon, with the use of a premeasured string, to the desired location for jejunal cannula placement. Using this technique allowed for modern distal ileal t-cannulation techniques to be employed, while alleviating the need for a midline laparotomy. However, one disadvantage was that the small intestine, prior to the intersection of the transverse spiral colon, was not measured, possibly affecting the consistency of jejunal t-cannula placement. A homogenous group of gilts with similar body weight were used to abate this effect. On day 67 , the mean jejunal t-cannula placement had a coefficient of variation of $3.33 \%$, suggesting this technique, in a homogenous group of pigs, resulted in consistent cannula placement.

The location and method for externalising t-cannulas can affect their longevity and function (Wubben et al., 2001). In order to externalise the second cannula on the same lateral side, the ileal $t$-cannula was externalised more dorsally to the initial laparotomy to accommodate the second t-cannula. Furthermore, the jejunal t-cannula was externalised in a location that would not interfere with ileal sample collections and was out of range of the hind limbs (Figure 3I). While externalising t-cannulas between the last two ribs has been previously reported, it is more invasive, limits the diameter of the barrel and reduces cannula longevity (Stein et al., 1998; Wubben et al., 2001). The abdominal wall, ventral to the ileal t-cannula location, is leaner and less robust, and thus requires a lighter weight $\mathrm{t}$-cannula with greater external reinforcement to prevent twisting. Two incidences of cannula torsion occurred with the jejunal cannula in this study, but after implementation of a secondary support washer to reinforce the abdominal wall, no other cases of torsion occurred.

The size and configuration of the jejunal and ileal t-cannulae were appropriate for the procedure described in this study for $30 \mathrm{~kg}$ pigs. Compared to the t-cannulas used in typical ileal cannulation procedures, those used in the duodenum or jejunum will require a shorter flange width, due to the reduced intestinal diameter. Likewise, when performing this procedure in pigs weighing less than $30 \mathrm{~kg}$, an even greater reduction in flange width may be required. The dimensions of the t-cannulas allowed digesta to flow freely. Jejunal digesta flow was quicker than ileal and ceased 6 hours post-feeding. This was anticipated due to reduced length of intestine before the jejunal t-cannula, and suggested that more cranial t-cannula placement can lead to reduced total collection time and increased collection frequency.

To accommodate growth, washers on both t-cannulas were adjusted at the end of each experimental period. Barrel length is a primary factor limiting t-cannula longevity. Accretion of tissue through growth reduces the length of exposed barrel and impairs collection bag attachment (Wubben et al., 2001). Experiments that need to collect digesta for a greater range of body weight should consider a t-cannula with a longer barrel.

\section{Conclusions}

Modifying the location of the initial laparotomy location, adjusting cannula externalisation sites and using the duodenum suspensory ligament and the point where the duodenum is posterior to the transverse colon as anatomical landmarks, modern ileal t-cannulation procedures can be modified to implement a proximal second $t$-cannula, with 
minimal variation. This modified procedure can be used to expand cannulation studies to evaluate digesta composition at multiple intestinal locations, without drastically altering commonly implemented surgical characteristics.

\section{Acknowledgements}

The authors would like to express appreciation to AB Vista Feed Ingredients and the Agriculture and Food Research Initiative Competitive Grant nos. 2016-38420-25496 from the United States Department of Agriculture National Institute of Food and Agriculture for financial support of this research.

\section{Conflict of interest}

The authors declare no conflict of interest.

\section{References}

Albin, D.M., Wubben, J.E. and Gabert, V.M., 1999. Approaches to collecting ileal digesta from swine. University of Illinois Extension, Champaign, IL, USA. Available at: http://livestocktrail.illinois.edu/ porknet/paperDisplay.cfm?ContentID=71.

Beale, C.N., Reyelt, L.A., Bogins, C.A., Chan, N.Y. and Perkins, S.E., 2018. Duodenal cannulation in pigs (Sus scrofa) as a drug delivery method. Comparative Medicine 68: 48-55.

Braude, R., Fulford, R.J. and Low, A.G., 1976. Studies on digestion and absorption in the intestines of growing pigs. Measurements of the flow of digesta and $\mathrm{pH}$. British Journal of Nutrition 36: 497-510. https://doi.org/10.1079/BJN19760104

FASS, 2010. Guide for the care and use of agricultural animals in research and teaching (Ag guide): third edition. Champaign, IL, USA, 177 pp. Available at: https://tinyurl.com/y52xlfgd.

Furuya, S., Takahashi, S. and Omori, S., 1974. The establishment of T-piece cannula fistulas into the small intestine of the pig. Nihon Chikusan Gakkaiho 45: 42-44. https://doi.org/10.2508/ chikusan.45.42

Gargallo, J. and Zimmermann, D.R., 1980. A simple intestinal cannula for swine. American Journal of Veterinary Research 41: 618-619.
Green, S., Bertrand, S.L., Duron, M.J.C. and Maillard, R.A., 1987. Digestibility of amino acids in maize, wheat and barley meal, measured in pigs with ileo-rectal anastomosis and isolation of the large intestine. Journal of the Science of Food and Agriculture 41: 29-43. https://doi.org/10.1002/jsfa.2740410104

Gutierrez, N.A., Serao, N.V.L. and Patience, J.F., 2016. Effects of distillers' dried grains with solubles and soybean oil on dietary lipid, fiber, and amino acid digestibility in corn-based diets fed to growing pigs. Journal of Animal Science 94: 1508-1519. https:// doi:10.2527/jas.2015-9529

Knabe, D.A., LaRue, D.C., Gregg, E.J., Martinez, G.M. and Tanksley, T.D., 1989. Apparent digestibility of nitrogen and amino acids in protein rich feedstuffs by growing pigs. Journal of Animal Science 67: 441-458. https://doi.org/10.2527/jas1989.672441x

Li, R., Hou, G.F., Song, Z.H., Zhao, J.F., Fan, Z.Y., Hou, D.X. and Hea, X., 2019. Nutritional value of enzyme-treated soybean meal, concentrated degossypolized cottonseed protein, dried porcine solubles and fish meal for 10 - to $20 \mathrm{~kg}$ pigs. Animal Feed Science and Technology 252: 23-33. https://doi.org/10.1016/j. anifeedsci.2019.04.002

NRC, 2012. Nutrient requirements of swine. $11^{\text {th }}$ revised edition. The National Academies Press, Washington, DC, USA, 400 pp.

Newman, M.A., Zebeli, Q., Velde, K., Grüll, D., Molnar, T., Kandler, W. and Metzler-Zebeli, B.U., 2016. Enzymatically modified starch favorably modulated intestinal transit time and hindgut fermentation in growing pigs. PloS ONE 11(12): e0167784. https:// doi.org/10.1371/journal.pone.0167784

Stein, H.H., Shipley, C.F. and Easter, R.A., 1998. Technical note: a technique for inserting a T-cannula into the distal ileum of pregnant sows. Journal of Animal Science 76: 1433-1436. https:// doi.org/10.2527/1998.7651433x

Swindle, M.M. and Smith, A.C., 2015. Swine in the laboratory: surgery, anesthesia, imaging, and experimental techniques. $3^{\text {rd }}$ edition. CRC press. Boca Raton, FL, USA, 594 pp.

Walker, W.R., Morgan, G.L. and Maxwell, C.V., 1986. Ileal cannulation in baby pigs with a simple T-cannula. Journal of Animal Science 62: 407-411. https://doi.org/10.2527/jas1986.622407x

Wubben, J.E., Smiricky, M.R., Albin, D.M. and Gabert, V.M., 2001. Improved procedure and cannula design for simple-T cannulation at the distal ileum in growing pigs. Laboratory Animal Science, 40: 27-31. 
